\title{
First report of human babesiosis in Australia
}

\section{Sanjaya N \\ Senanayake \\ MB BS, FRACP, MAppEpid, Associate Professor of Medicine,' and Infectious Diseases Physician \\ Andrea Paparini $\mathrm{MSc}, \mathrm{PhD}$ Research Fellow, School of Veterinary and Biomedical Sciences \\ Maya Latimer MB BS, FRACP, FRCPA Haematologist, Department of Haematology ${ }^{2}$ \\ Kerrie Andriolo GradCert (DiagnosticPathology) Chief Scientist, Department of Haematology}

Alexandre J Dasilva PhD,
logist Research Microbiologist

Heather Wilson MB BS, PhD, Medical Officer ${ }^{2}$

Maniphet V Xayavong BA, MBA, Scientist ${ }^{4}$

Peter J Collignon MBBS, FRACP, FRCPA Professor of Medicine and Infectious Diseases Physician $^{2}$

Phillip Jeans MB BS, FRACS, Surgeon Department of Surgery 2

Peter J Irwin BVetMed, PhD, FANZCVSc Associate Professor, School of Veterinary and Biomedical Sciences ${ }^{3}$

1 Australian National University, Canberra, ACT.

2 Canberra Hospital, Canberra, ACT.

3 Murdoch University, Perth, WA.

4 Centers for Diseas Control and Prevention Atlanta, Ga, USA

sanjaya.senanayake@ act.gov.au

MJA 2012; 196: 350-352 doi: 10.5694/mjall.11378

Clinical focus p 322 Research p 345

\section{We report the first human case of babesiosis in Australia, thought to be locally acquired}

\section{Clinical record}

A 56-year-old man with serious hepatic, renal and bony injuries was transferred to Canberra Hospital in mid November 2010 following a motor vehicle accident. His medical history included type 1 diabetes mellitus, excessive alcohol and prescription medicine use, depression, hypertension and hypercholesterolaemia. He had no history of injecting drug use. He had been living on the south coast of New South Wales with his son.

During the first 4 months of his admission he required surgery, prolonged broad-spectrum antibiotic therapy and total parenteral nutrition. He developed worsening cholestatic liver function, moderate-to-severe thrombocytopenia and fluctuating anaemia. By late March 2011, he was pancytopenic with worsening anaemia due to intravascular haemolysis, lymphopenia and severe thrombocytopenia, and required ongoing blood product transfusions. The result of an HIV test was negative.

In early April, the patient was transferred to the intensive care unit because of respiratory and haemodynamic deterioration. On 10 April, intraerythrocytic parasites were incidentally identified during routine examination of blood films taken that morning. Ringform parasites, initially thought to be a Plasmodium species, were confirmed on thick and thin films. Results of immunochromatographic tests were negative for Plasmodium falciparum; however, intravenous artesunate and primaquine therapy for presumed severe malaria was commenced. Broad-spectrum antibiotic therapy was continued. After 48 hours without clinical improvement, the parasitaemia level had increased from $1.7 \%$ to $2.6 \%$ infected red blood cells. Re-examination of the blood films and recognition that the organisms did not produce hemozoin led to the presumptive diagnosis of babesiosis infection. This was confirmed by Australian and overseas experts who viewed electronic files of the slides. The patient was then given intravenous quinine $(600 \mathrm{mg} 8$ hourly) and clindamycin (600 mg 6-hourly) for babesiosis. Azithromycin and tigecycline were also added to provide additonal broad-spectrum antimicrobial cover, but clinical deterioration continued, and the levels of parasitaemia, anaemia and thrombocytopenia did not improve. He developed multiorgan failure and required haemodialysis. The parasitaemia peaked at $5.1 \%$ infected red blood cells despite ongoing blood transfusions (including 18 units of packed red cells over 8 days).
As his condition was too unstable for him to undergo total red cell exchange, an exchange of $500 \mathrm{~mL}$ of blood was performed on 16 April. He did not recover from the multiorgan failure, and severe thrombocytopenia contributed to acute gastrointestinal bleeding. On 18 April - 5 days after commencing specific antibabesiosis therapy - he suffered a fatal asystolic arrest.

Following the patient's death, a retrospective study of the blood films taken during his hospital admission was undertaken. Ring-form parasites were detected in very low numbers back to the end of the first week of March 2011, coinciding with the development of severe thrombocytopenia.

Initially, the multiple blood products that the patient received during his admission were thought to be the likely cause of his babesiosis. But blood films stored by his local pathology service and taken between September and November 2010 (ie, before his hospital admission) revealed pre-existence of the intraerythrocytic parasite and hyposplenic features. As the patient had not received blood products before his hospital admission in November 2010, the parasitaemia could not have been transfusion related. He had reported being bitten by ticks, but had been too sick to qualify this further. Due to general ill health, he had not worked or left the local area for many years. His only overseas travel had been to New Zealand almost 40 years earlier, a country with no known human babesiosis. It thus seemed unlikely that the babesiosis was acquired from overseas.

The patient had lived on the south coast of New South Wales for over 30 years - on a small farm with two horses and two Staffordshire bull terriers for 25 years, and then at a house in a small town with his son and a new Staffordshire bull terrier (acquired from within Australia) for 8 years. The new Staffordshire bull terrier was their only pet and was still living at their south coast home in mid 2011. Both his son (who was asymptomatic) and his Staffordshire bull terrier underwent testing for babesiosis; the results were negative.

To identify the organism and further elucidate its source, blood samples and films from the patient were examined at the Centers for Disease Control and Prevention (CDC) in Atlanta, United States, and at the School of Veterinary and Biomedical Sciences, Murdoch University, Western Australia. Both laboratories agreed that the morphological characteristics of the parasites in 
1 Blood film from a 56-year-old man infected with Babesia microti, showing a tetrad form (black arrow) and single ovoid forms (white arrows)

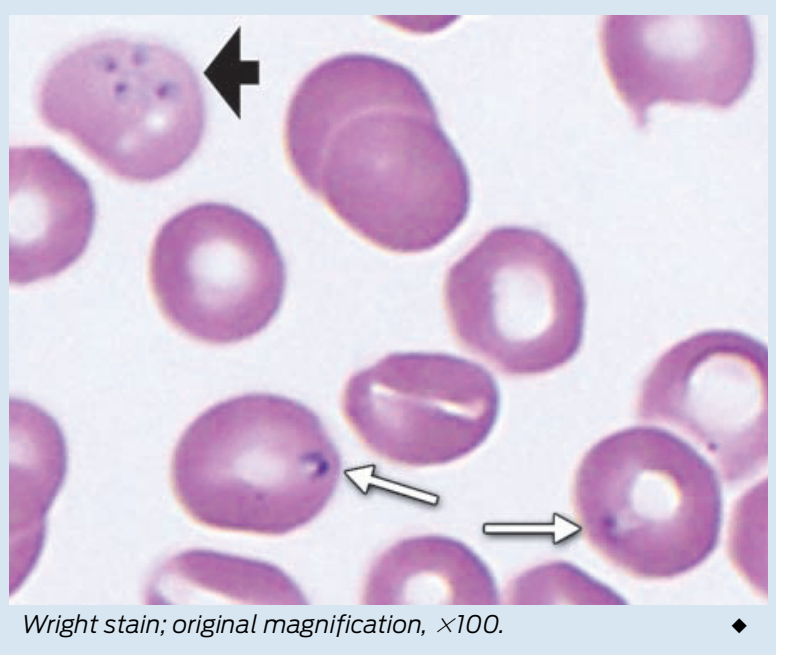

the blood films were consistent with a small Babesia. Intraerythrocytic organisms were mostly single and measured 1.5-2.5 $\mu \mathrm{m}$ in size but were highly polymorphic; pyriform and ovoid forms predominated, bizarre amoeboid forms were also common, and an occasional tetrad (Maltese cross form) was noted (Box 1). Immunofluorescent antibody testing for Babesia microti was performed by the CDC on serum samples from the patient (positive result, with titre of $1: 256$ ) and his son (negative result).

Complete sequencing of the $18 \mathrm{~S}$ ribosomal RNA gene (18S rDNA) and partial sequencing of the $\beta$-tubulin gene (both amplified by polymerase chain reaction) confirmed that the organism in the patient's blood was B. microti. At the CDC, a nested PCR that specifically amplifies a 154base-pair fragment from the $B$. microti $18 \mathrm{~S}$ rDNA was initially used to confirm the presence of B. microti in the patient's blood. In addition, sequencing of a 1767-basepair fragment amplified with primers Crypto FL (5'AACCTGGTTGATCCTGCCAGTAGTCAT-3') and Crypto RN (5'-GAATGATCCTTCCGCAGGTTCACCTAC-3'), was performed to strengthen the PCR findings. ${ }^{1}$ The $18 \mathrm{~S}$ rDNA sequence obtained was $100 \%$ similar to the GenBank entry AY693840 obtained from a B. microti isolate $18 \mathrm{~S}$ rDNA gene. At Murdoch University, two nested sets of universal piroplasm 18S rDNA primers were used, one of which has been published. ${ }^{2}$ The patient's son's blood was used as a negative control. The consensus sequence was $100 \%$ homologous to known human-derived Babesia species isolates. In addition, five novel primer sets designed during this study were used to obtain a partial $\beta$-tubulin gene fragment (791 base pairs), which confirmed the presence of $B$. microti in the patient's blood and showed 100\% homology with North American isolates (eg, GenBank entries AB083377 and AY144722). A phylogenetic tree for this locus (produced using the maximum likelihood method) revealed clustering with isolates of $B$. microti obtained from the tick species Ixodes scapularis (formerly known as Ixodes dammini), humans and voles in North America.

\section{Discussion}

To our knowledge, this is the first report of a human case of babesiosis in Australia, which we believe was locally acquired.

Human babesiosis is an emerging tick-borne zoonosis. The first human case was reported in Croatia in $1957 .{ }^{3}$ In 1968, Babesia divergens was identified as the cause of human babesiosis in Europe; this was soon followed by the discovery of human cases of $B$. microti infection in the US. ${ }^{4}$ More recently, human cases of babesiosis have emerged from Asia, Africa and South America. ${ }^{5-8}$

Since B. microti has never been detected in Australia before, its discovery as the cause of infection in this patient, who had no significant history of travel, raises intriguing questions about its natural hosts and epidemiology on this continent. Traditionally, B. microti is considered to have a Holarctic distribution, associated with a variety of small mammalian hosts (rodents, including voles, and shrews), and is transmitted by several Ixodes tick species present throughout the northern hemisphere, with humans becoming infected as accidental hosts. Recent phylogenetic analyses based on complete sequences of the genes encoding $18 \mathrm{~S}$ ribosomal RNA, $\beta$ tubulin and the $\eta$ subunit of the chaperonin-containing t-complex polypeptide 1 suggest that $B$. microti represents a genetically diverse species complex that comprises several geographically distinct clusters located in North America, Eurasia and Japan, and is closely related to "Babesia microti-like" species isolated from an everexpanding range of feral and domesticated mammal hosts. ${ }^{9}$

In Australia, babesiosis is a well documented disease of cattle (Babesia bigemina and Babesia bovis) and dogs (Babesia canis, Babesia vogeli and Babesia gibsoni), and babesiosis tick vectors have been imported to the continent since European settlement. ${ }^{10,11}$ Australia also has a diverse variety of native Ixodes ticks, including Ixodes holocyclus (responsible for tick paralysis), and a few Babesia and Theileria species have been described morphologically in native marsupial hosts (but not $B$. microti). ${ }^{12}$ Unfortunately, a paucity of molecular studies means that the taxonomy and phylogenetic relationships

\section{Diagnosing human babesiosis in Australia \\ When to suspect babesiosis \\ Clinicians should suspect babesiosis in patients in Australia who have haemolytic anaemia, thrombocytopenia, fever, an influenza-like illness and a history of at least one of the following: \\ - tick bites \\ outdoor activities putting one at risk of tick bites \\ - transfusion of blood products \\ - overseas travel to a region where babesiosis is endemic.}

How to proceed with the diagnosis

Thick and thin blood films should be examined for intraerythrocytic parasites (three sets of films should be taken, 8-12 hours apart). If the results of blood films are negative but the diagnosis is still suspected, antibody testing of serum and molecular testing of blood (by polymerase chain reaction) can be done. 
of the endemic piroplasms (intraerythrocytic tick parasites, including Babesia and Theileria) are not well understood.

Based on phylogenetic analysis, the isolate from this patient was most closely related to North American strains of B. microti, so it is unlikely that the piroplasm described here originated from a native Australian mammal, but not impossible. In the absence of transfusion or injecting drug history, the patient must have become infected following a tick bite. Two scenarios seem probable. The patient might have been bitten by an imported tick (contained within clothing or luggage that had recently arrived from an endemic country), but no history suggested such contact. Alternatively, a local tick might have transmitted an autochthonous infection, presumably originating from one or more species of introduced rodent.

The natural history of this patient's infection is notable. Babesia ring forms were detectable on routine blood films taken while he was an outpatient -7 months before he died. At that time, he was asymptomatic and neither anaemic nor thrombocytopenic, but 6 months later the babesiosis became symptomatic and severe. The parasitaemia of $5.1 \%$ around the time of his death was likely to have been an underestimate of the true figure as it would have been diluted by the multiple blood products that he was receiving at the time. Asymptomatic parasitaemia is well described in babesiosis, both in the setting of primary infection and following treatment of symptomatic infection. ${ }^{4}$ It is unclear what transformed this patient's chronic asymptomatic infection into a severe symptomatic infection that probably contributed to his death. He did have risk factors for severe babesiosis: hyposplenism, liver impairment and his age; ${ }^{4}$ however, these were present when the infection was asymptomatic months earlier. It is possible that his chronic hospitalisation, and general deconditioning from the long admission, resulted in significant immunosuppression.

Severe babesiosis from B. microti is a serious condition with a case fatality rate of $5 \%-10 \% .^{13}$ Indeed, this patient's condition did not improve despite his receiving recommended therapy once the diagnosis of babesiosis was made. Even the artesunate that he received for suspected malaria (immediately before the diagnosis) has been shown to have activity against B. microti in animal models. ${ }^{14}$

Although the animal host for B. microti is yet to be identified in Australia, the proximity of ticks, other wildlife and human populations along Australia's eastern seaboard means that further cases may be encountered. Clinicians working in Australia should therefore be aware of the signs and symptoms of babesiosis and how to diagnose it (Box 2). Further investigation into the piroplasms of native mammals, introduced rodents and their ticks is necessary to identify the source of this infection. As transfusion-related babesiosis is well recognised in other countries, ${ }^{15}$ this case may have future implications for the screening of blood products in Australia.

Acknowledgements: We thank Michael Pidcock, Grace Moshi and Rachel Murray (Canberra Hospital) for their haematological expertise and Bert de Vos (Biosecurity Queensland) for his advice on bovine babesiosis.

Competing interests: No relevant disclosures.

Received 27 Oct 2011, accepted 5 Feb 2012

1 Herwaldt BL, Cacciò S, Gherlinzoni F, et al. Molecular characterization of a non-Babesia divergens organism causing zoonotic babesiosis in Europe. Emerg Infect Dis 2003; 9: 942-948.

2 Jefferies R, Ryan UM, Irwin PJ. PCR-RFLP for the detection and differentiation of the canine piroplasm species and its use with filter paper-based technologies. Vet Parasitol 2007; 144: 20-27.

3 Skrabalo Z, Deanovic Z. Piroplasmosis in man; report of a case. Doc Med Geogr Trop 1957; 9: 11-16.

4 Vannier E, Gewurz BE, Krause PJ. Human babesiosis. Infect Dis Clin North Am 2008; 22: 469-488.

$5 \mathrm{Kim} \mathrm{JY}, \mathrm{Cho} \mathrm{SH}$, Joo HN, et al. First case of human babesiosis in Korea: detection and characterization of a novel type of Babesia sp. (KOI) similar to ovine babesia. J Clin Microbiol 2007; 45: 2084-2087.

6 Bush JB, Isaäcson M, Mohamed AS, et al. Human babesiosis-a preliminary report of 2 suspected cases in South Africa. S Afr Med J 1990; 78: 699.

7 Rios L, Alvarez G, Blair S. Serological and parasitological study and report of the first case of human babesiosis in Colombia. Rev Soc Bras Med Trop 2003; 36: 493-498.

8 Marathe A, Tripathi J, Handa V, Date V. Human babesiosis-a case report. Indian JMed Microbiol 2005; 23: 267-269.

9 Nakajima R, Tsuji M, Zamoto-Niikura A, et al. Babesia microti-group parasites compared phylogenetically by complete sequencing of the CCTeta gene in 36 isolates. J Vet Med Sci 2009; 71: 55-68.

10 Bock R, Jackson L, de Vos A, Jorgensen W. Babesiosis of cattle. Parasitology 2004; 129 Suppl: S247-S269.

11 Jefferies R, Ryan UM, Jardine J, et al. Blood, bull terriers and babesiosis: further evidence for direct transmission of Babesia gibsoni in dogs. Aust Vet J 2007; 85: 459-463.

12 Clark P. Haematology of Australian mammals. 1st ed. Melbourne: CSIRO Publishing, 2004.

13 Hatcher JC, Greenberg PD, Antique J, Jimenez-Lucho VE. Severe babesiosis in Long Island: review of 34 cases and their complications. Clin Infect Dis 2001; 32: $1117-1125$.

14 Goo YK, Terkawi MA, Jia H, et al. Artesunate, a potential drug for treatment of Babesia infection. Parasitol Int 2010; 59: 481-486.

15 Leiby DA. Transfusion-transmitted Babesia spp.: bull's-eye on Babesia microti. Clin Microbiol Rev 2011; 24: 14-28. 


\section{Case reports}

